
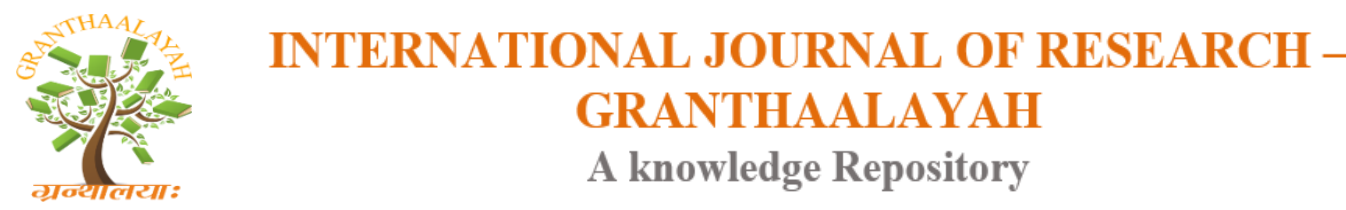

Science

\title{
ANALYSES OF PARTICULATE MATTER AT CONSTRUCTION SITES: CASE STUDY OF OBIO-AKPO LOCAL GOVERNMENT AREA, RIVERS STATE, NIGERIA
}

\author{
Robert J.J ${ }^{* 1}$, Chineke T.C ${ }^{2}$, Chiemeka I.U ${ }^{3}$ \\ ${ }^{*}$ Department of Physics, Ignatius Ajuru University of Education, Rumuolumeni, Port-Harcuourt \\ ${ }^{2}$ Department of Physics, Imo State University, Owerri \\ ${ }^{3}$ Department of Physics, Abia State University, Uturu
}

\begin{abstract}
This study focused on analyses of particulate matter generated from construction at some selected building sites in Obio-Akpo Local Government Area (latitudes $4^{\circ} 45^{\prime} \mathrm{N}$ and $4^{\circ} 60^{\prime} \mathrm{N}$ and longitudes $6^{\circ} 50^{\prime} \mathrm{E}$ and $8^{\circ} 00^{\prime} \mathrm{E}$ ) Nigeria. The method of sample collection was direct deposition method under gravity. The masses of particulate matter collected from two construction sites (sampling sites 1 and 2) and a residential building for sampling site 3(control) for a period of three weeks and two days (April 19th - May 10th, 2017) were 0.06g and 0.04g for sampling sites land 2, while sampling site 3 being the control was 0.02g. The Atomic Absorption Spectroscopic analyses of the particulate matter samples revealed the presence of thirty three (33) elemental components which include; beryllium(Be), sodium (Na), magnesium( $\mathrm{Mg})$, aluminium( $(\mathrm{Al}), \operatorname{potassium}(\mathrm{K}), \operatorname{calcium}(\mathrm{Ca}), \operatorname{vanadium}(\mathrm{V}), \operatorname{chromium}(\mathrm{Cr} 52 \& \mathrm{Cr} 53)$, manganese $(\mathrm{Mn})$, iron (Fe), cobalt(Co), nickel(Ni), copper(Cu), zinc(Zn), $\operatorname{arsenic}(\mathrm{As})$, molybdenum(Mo 95,\& Mo 98), cadmium(Cd106,Cd108 \& Cd 111), silver(Ag), indium(In), tin $(\mathrm{Sn})$, antimony $(\mathrm{Sb})$, barium $(\mathrm{Ba})$, terbium $(\mathrm{Tb})$, thallium(Tl), lead $(\mathrm{Pb})$, bismuth $(\mathrm{Bi})$, thorium(Th), and uranium(U). A good number of research works have shown that some of these elements constitute serious health problems to man when they are inhaled or ingested, hence the need for proper safety mechanisms to be put in place in order to protect construction workers and individuals living within the environment from their harmful effects.
\end{abstract}

Keywords: Analyses; Particulate Matter; Construction Sites.

Cite This Article: Robert J.J, Chineke T.C, and Chiemeka I.U. (2018). "ANALYSES OF PARTICULATE MATTER AT CONSTRUCTION SITES: CASE STUDY OF OBIO-AKPO LOCAL GOVERNMENT AREA, RIVERS STATE, NIGERIA." International Journal of Research - Granthaalayah, 6(1), 48-55. https://doi.org/10.29121/granthaalayah.v6.11.2018.1593. 


\section{Introduction}

Man's quality of life and survival on this planet Earth is partly tied to how clean the environment he lives in. Air pollution has been identified as one of the major causes of deaths around the world.

Research studies have shown that outdoor air pollution caused 3 million premature deaths yearly around the world and particulate matter was identified as the biggest culprit (Harvey, 2015). The International Agency for Research on Cancer categorized airborne particulates as a Group 1 carcinogen (Loomis et al, 2014). According to Gray (2017), construction industry contributes about $4 \%$ of particulate emissions, and is responsible for air, water and soil pollution. In the month of February, 2017, Rivers state government shut down a Chinese construction company (CGC) for producing thick black smog from their Asphalt plant (Daily Post February 11 ${ }^{\text {th }}, 2017$ ).

\subsection{Particulate (PM) Matter}

The United states Environmental Protection Agency (USEPA, 2017) defines particulate matter or particle pollution as a complex mixture of extremely small particles and liquid droplets that get into air. According to GreenFaacts (2017), particulate matter is predominantly made up of fine particles and its airborne total mass ranging from 0.1 to $2.5 \mu \mathrm{m}$ and the coarse fraction contains the larger particles with a size ranging from 2.5 to $10 \mu \mathrm{m}$.

\subsection{Sources of Particulate (PM) Matter}

The source of particulate matter could be natural or anthropogenic (man-made).

\subsubsection{Natural Source}

Volcanic eruptions, forest fires and sea spray, and dust particles carried by winds.

\subsubsection{Anthropogenic Source}

A number of humans activities such as; burning of fossils for energy production, road and building constructions, and farming lift microscopic particles into the Earth's atmosphere.

\subsection{Components of Particulate Matter}

Particulate matter components include sulphate, organic matter, black carbon, dust, pollen, smoke, liquid droplets and salt. According to Europeans Environmental Agency (EEA, 2012), sea salt consists of sodium chloride with traces of magnesium and sulphate.

\subsection{Effects of Particulate matter}

Particulate matter poses some health problems (such as lung cancer, asthma, deteriorating lung function, heart diseases, bronchitis and death in very serious cases) to man (Morawska, 1999). It also has some negative effects on the environment which include poor visibility, change in atmospheric properties, formation of fog and precipitation, damage to vegetation and materials 
and change in climate due to alteration of solar radiation, wind distribution and temperature (Morawska, 1999).

\section{Materials and Methods}

\subsection{The Study Area}

Obi-Apko Local Government Area of Rivers state is bounded by Port Harcourt local government area to the south, Ikwerre to the north, Oyigbo to the east, and Emohua to the west. It has a geographical location of latitudes $4^{\circ} 45^{\prime} \mathrm{N}$ and $4^{\circ} 60^{\prime} \mathrm{N}$ and longitudes $6^{\circ} 50{ }^{\prime} \mathrm{E}$ and $8^{\circ} 00^{\prime} \mathrm{E}$. It lies in the tropical wet climate zone, characterized by abundant rainfall with short dry season. The monsoon season occurs between April and October, bringing heavy rainfall ranging from 2000 to $2500 \mathrm{~mm}$ with temperatures up to $25^{\circ} \mathrm{C}$ and a relatively constant humidity (en.m.wikipedia.org/wiki/Obio-Akpor). Three sampling sites were chosen for the study; namely Rumuolumeni, Egbelu, and Ada-george. Rumuolumeni and Egbelu served as experiment while Ada-george was the controlled experiment.

\subsection{Collection of Sample}

The method of sample collection was direct deposition method under gravity using plastic funnel with Whatman filter paper of diameter $0.125 \mathrm{~m}$. For each of the construction sites, the funnel with Whatman filter paper was mounted 2 metres above the earth's surface and 10 metres away from the construction sites within Rumuolumeni and Egbelu which serve as the experiment. The third funnel with Whatman filter paper was mounted at a residential building at Ada-george, which is the control and a location of about 2kilometres from the construction sites.

The funnels were left for a period of three weeks and two days $\left(19^{\text {th }}\right.$ April $-11^{\text {th }}$ May, 2017) to collect the sample by direct deposition under gravity.

Some atmospheric variables for the period under study were measured using the following instruments; microprocessor Am-4832-digital anemometer, hygrometer, digital thermometer, rain gauge and digital electronic balance.

\subsection{Sample Analysis}

Atomic Absorption Spectroscopy (AAS) was employed in the analyses of the particulate matter samples collected. According to TutorVista.com (2017), AAS is a technique for measuring the concentration of various elements in the sample through their absorption of light. It is a relatively simple and reliable technique which uses absorption of optical radiation by free atoms for determining the contents of different element.

\section{Results and Discussion}

The masses of particulate matter measured for the period under study is shown in Tables 1 . 
Table1: Mass of particulate matter

\begin{tabular}{|l|c|c|c|}
\hline Sampling points & MFP(gm) & (MFP + MS) (gm) & MS(gm) \\
\hline Rumuolumeni (sampling site 1 -Experiment) & 1.08 & 1.49 & 0.06 \\
\hline Egbelu (sampling site 2 -Experiment) & 1.08 & 1.12 & 0.04 \\
\hline Ada-george (Sampling site 3 - Control) & 1.08 & 1.10 & 0.02 \\
\hline
\end{tabular}

\section{Mass of filter paper $=$ MFP}

Mass of sample $\quad=$ MS

The masses of particulate matter collected from two construction sites (sampling sites 1 and 2) and a residential building for sampling site 3(control) for a period of three weeks and two days (April $19^{\text {th }}-$ May $10^{\text {th }}, 2017$ ) are $0.06 \mathrm{~g}$ and $0.04 \mathrm{~g}$ for sampling sites 1 and 2 respectively, while sampling site 3 being the control is $0.02 \mathrm{~g}$.

\subsection{Particulate Matter Analyses}

Table 2: Sample Solution for analyses

\begin{tabular}{|l|c|c|c|}
\hline Sampling points & $\begin{array}{c}\text { Weight of the bottle } \\
\text { before digestion }\end{array}$ & $\begin{array}{c}\text { Weight of the bottle } \\
\text { after digestion }\end{array}$ & $\begin{array}{c}\text { Water } \\
\text { (g) }\end{array}$ \\
\hline & {$[\mathrm{g}]$} & {$[\mathrm{g}]$} & {$[\mathrm{g}]$} \\
\hline Rumuolumeni(Sampling site 1) & 11.27 & 42.25 & 30.98 \\
\hline Egbelu(Sampling site 2) & 11.29 & 42.5 & 31.21 \\
\hline Adageorge(Sampling site 3) & 11.20 & 41.99 & 30.79 \\
\hline
\end{tabular}

Elemental components of the particulate matter sample from Atomic Absorption Spectroscopy (AAS) analyses are shown in Table 4.

Table 3: Elemental components of the particulates and their concentration in microgram per metre cube $(\mu \mathrm{g} / \mathrm{m} 3)$

\begin{tabular}{|c|c|c|c|c|c|c|c|c|c|c|}
\hline & $\begin{array}{l}\text { Weight of } \\
\text { particulat } \\
\text { e matter }\end{array}$ & $\begin{array}{l}(1) \\
\\
\text { Be } \\
/ 9\end{array}$ & $\begin{array}{l}\text { (2) } \\
\mathrm{Na} / \\
23\end{array}$ & $\begin{array}{l}\text { (3) } \\
\mathrm{Mg} / 24\end{array}$ & $\begin{array}{l}\text { (4) } \\
\mathrm{Al} / 27\end{array}$ & $\begin{array}{l}\text { (5) } \\
\text { K / } 39\end{array}$ & $\begin{array}{l}\text { (6) } \\
\mathrm{Ca} / 43\end{array}$ & $\begin{array}{l}\text { (7) } \\
\text { V / } 51\end{array}$ & $\begin{array}{l}\text { (8) } \\
\mathrm{Cr} / 52\end{array}$ & $\begin{array}{l}\text { (9) } \\
\mathrm{Cr} \\
\mathbf{5 3}\end{array}$ \\
\hline $\begin{array}{l}\text { Sampling } \\
\text { points }\end{array}$ & [g] & $\begin{array}{l}\text { Co } \\
\text { nc. } \\
{[} \\
\mu \mathrm{g} / \\
\left.\mathrm{m}^{3}\right]\end{array}$ & $\begin{array}{l}\text { Conc } \\
\dot{[} \\
{[\mu \mathrm{g} /} \\
\left.\mathrm{m}^{3}\right]\end{array}$ & $\begin{array}{l}\text { Conc. } \\
{\left[\mu \mathrm{g} / \mathrm{m}^{3}\right]}\end{array}$ & $\begin{array}{l}\text { Conc. } \\
{\left[\mu \mathrm{g} / \mathrm{m}^{3}\right]}\end{array}$ & $\begin{array}{l}\text { Conc. } \\
{\left[\mu \mathrm{g} / \mathrm{m}^{3}\right]}\end{array}$ & $\begin{array}{l}\text { Conc. } \\
{\left[\begin{array}{l}\left.\mathrm{m}^{3}\right] \\
\mathrm{g} /\end{array}\right.}\end{array}$ & $\begin{array}{l}\text { Conc. } \\
{\left[\begin{array}{l}\left.\mathrm{m}^{3}\right] \\
\mathrm{g} / \mathrm{g} /\end{array}\right.}\end{array}$ & $\begin{array}{l}\text { Conc. } \\
{\left[\begin{array}{l}\left.\mathrm{m}^{3}\right] \\
\left.{ }^{3}\right]\end{array}\right.}\end{array}$ & $\begin{array}{l}\text { Conc. } \\
{\left[{ }_{\left.\mathrm{m}^{3}\right]}^{\mu \mathrm{g} /}\right.}\end{array}$ \\
\hline $\begin{array}{l}\text { Rumuolu } \\
\text { meni }\end{array}$ & 0.037 & $\begin{array}{l}927 \\
.81\end{array}$ & $\begin{array}{l}4733 \\
908 . \\
00\end{array}$ & $\begin{array}{l}1029153 \\
0.00\end{array}$ & $\begin{array}{l}6561870 \\
0.00\end{array}$ & $\begin{array}{l}1283220 \\
0.00\end{array}$ & $\begin{array}{l}108291 \\
6.00\end{array}$ & $\begin{array}{l}139860 \\
.00\end{array}$ & $\begin{array}{l}167388 \\
.00\end{array}$ & $\begin{array}{l}19101 \\
.00\end{array}$ \\
\hline Egbelu & 0.045 & $\begin{array}{l}330 \\
.75\end{array}$ & $\begin{array}{l}2936 \\
682 . \\
00\end{array}$ & $\begin{array}{l}4613564 . \\
00\end{array}$ & $\begin{array}{l}6182800 \\
0.00\end{array}$ & $\begin{array}{l}6670742 . \\
00\end{array}$ & $\begin{array}{l}244811 . \\
90\end{array}$ & $\begin{array}{l}117086 \\
.00\end{array}$ & $\begin{array}{l}153871 \\
.00\end{array}$ & $\begin{array}{l}17682 \\
.00\end{array}$ \\
\hline $\begin{array}{l}\text { Adageorg } \\
\mathrm{e}\end{array}$ & 0.01 & $\begin{array}{l}54 . \\
07\end{array}$ & $\begin{array}{l}1047 \\
293 . \\
00\end{array}$ & $\begin{array}{l}528389.7 \\
0\end{array}$ & $\begin{array}{l}6802969 . \\
00\end{array}$ & $\begin{array}{l}1419079 . \\
00\end{array}$ & $\begin{array}{l}25007.5 \\
0\end{array}$ & $\begin{array}{l}16392 . \\
08\end{array}$ & $\begin{array}{l}31596 . \\
00\end{array}$ & $\begin{array}{c}3074 . \\
00\end{array}$ \\
\hline
\end{tabular}




\begin{tabular}{|c|c|c|c|c|c|c|c|c|c|}
\hline & $\begin{array}{l}\text { Weight } \\
\text { of } \\
\text { particul } \\
\text { ate } \\
\text { matter }\end{array}$ & $\begin{array}{l}\text { (10) } \\
\text { Mn / } 55\end{array}$ & $\begin{array}{l}\text { (11) } \\
\mathrm{Fe} / 56\end{array}$ & $\begin{array}{l}\text { (12) } \\
\text { Co / } 59\end{array}$ & $\begin{array}{l}\text { (13) } \\
\mathrm{Ni} / 60\end{array}$ & $\begin{array}{l}(14) \\
\mathrm{Cu} / 63\end{array}$ & $\begin{array}{l}\text { (15) } \\
\mathrm{Zn} / 66\end{array}$ & $\begin{array}{l}(16) \\
\text { As } \\
75\end{array}$ & $\begin{array}{l}\text { (17) } \\
\text { Mo / } \\
95\end{array}$ \\
\hline $\begin{array}{l}\text { Sampling } \\
\text { points }\end{array}$ & [g] & $\begin{array}{l}\text { Conc. } \\
{\left[\mu \mathrm{g} / \mathrm{m}^{3}\right]}\end{array}$ & $\begin{array}{l}\text { Conc. } \\
{\left[\mu \mathrm{g} / \mathrm{m}^{3}\right]}\end{array}$ & $\begin{array}{l}\text { Conc. } \\
{\left[\mu \mathrm{g} / \mathrm{m}^{3}\right]}\end{array}$ & $\begin{array}{l}\text { Conc. } \\
{\left[\begin{array}{ll}{[} & \mu \mathrm{g} / \\
\left.\mathrm{m}^{3}\right]\end{array}\right.}\end{array}$ & $\begin{array}{l}\text { Conc. } \\
{\left[\begin{array}{ll}{ }^{3} & \mu \mathrm{g} / \\
\left.\mathrm{m}^{3}\right]\end{array}\right.}\end{array}$ & $\begin{array}{l}\text { Conc. } \\
{\left[\mu \mathrm{g} / \mathrm{m}^{3}\right]}\end{array}$ & $\begin{array}{l}\text { Conc. } \\
{[\mu \mathrm{g} /} \\
\left.\mathrm{m}^{3}\right]\end{array}$ & $\begin{array}{l}\text { Conc. } \\
{\left[{ }_{\left.\mathrm{m}^{3}\right]}^{\mu \mathrm{g} /}\right.}\end{array}$ \\
\hline $\begin{array}{l}\text { Rumuolum } \\
\text { eni }\end{array}$ & 0.037 & $\begin{array}{l}1810353.0 \\
0\end{array}$ & $\begin{array}{l}204977400 . \\
00\end{array}$ & $\begin{array}{l}110614 . \\
00\end{array}$ & $\begin{array}{l}18084 . \\
00\end{array}$ & $\begin{array}{l}60632 . \\
00\end{array}$ & $\begin{array}{l}116679.0 \\
0\end{array}$ & $\begin{array}{l}814.0 \\
0\end{array}$ & $\begin{array}{l}2179.0 \\
0\end{array}$ \\
\hline Egbelu & 0.045 & $\begin{array}{l}1945338.0 \\
0\end{array}$ & $\begin{array}{l}141809600 . \\
00\end{array}$ & $\begin{array}{l}101285 . \\
00\end{array}$ & $\begin{array}{l}13688 . \\
00\end{array}$ & $\begin{array}{l}68680 . \\
00\end{array}$ & $\begin{array}{l}153803.0 \\
0\end{array}$ & $\begin{array}{l}680.0 \\
0\end{array}$ & $\begin{array}{l}1914.0 \\
0\end{array}$ \\
\hline Adageorge & 0.01 & 375938.00 & $\begin{array}{l}100303100 . \\
00\end{array}$ & $\begin{array}{l}10647.0 \\
0\end{array}$ & $\begin{array}{l}3475.0 \\
0\end{array}$ & $\begin{array}{l}11139 . \\
00\end{array}$ & $\begin{array}{l}1024980 . \\
00\end{array}$ & $\begin{array}{l}169.0 \\
0\end{array}$ & 398.00 \\
\hline
\end{tabular}

\begin{tabular}{|c|c|c|c|c|c|c|c|c|}
\hline & $\begin{array}{l}\text { Weight of } \\
\text { particulate } \\
\text { matter }\end{array}$ & $\begin{array}{l}\text { (18) } \\
\text { Mo / } 98\end{array}$ & $\begin{array}{l}\text { (19) } \\
\text { Cd }\end{array}$ & $\begin{array}{l}\text { (20) } \\
\text { Ag } \\
107\end{array}$ & $\begin{array}{l}\text { (21) } \\
\text { Cd / } 108\end{array}$ & $\begin{array}{l}\text { (22) } \\
\text { Cd / } 111\end{array}$ & $\begin{array}{l}\text { (23) } \\
\text { In / } 115\end{array}$ & $\begin{array}{l}(24) \\
\text { Sn / } 118\end{array}$ \\
\hline $\begin{array}{l}\text { Sampling } \\
\text { points }\end{array}$ & [g] & $\begin{array}{l}\text { Conc. } \\
{\left[\mu \mathrm{g} / \mathrm{m}^{3}\right]}\end{array}$ & $\begin{array}{l}\text { Conc. } \\
{\left[\begin{array}{l}\left.\mathrm{m}^{3}\right] \\
\mathrm{g}\end{array}\right.}\end{array}$ & $\begin{array}{l}\text { Conc. } \\
{\left[\mu \mathrm{g} / \mathrm{m}^{3}\right]}\end{array}$ & $\begin{array}{l}\text { Conc. } \\
{\left[\mu \mathrm{g} / \mathrm{m}^{3}\right]}\end{array}$ & $\begin{array}{l}\text { Conc. } \\
{\left[\mu \mathrm{g} / \mathrm{m}^{3}\right]}\end{array}$ & $\begin{array}{l}\text { Conc. } \\
{\left[\mu \mathrm{g} / \mathrm{m}^{3}\right]}\end{array}$ & $\begin{array}{l}\text { Conc. } \\
{\left[\mu \mathrm{g} / \mathrm{m}^{3}\right]}\end{array}$ \\
\hline Rumuolumeni & 0.037 & 3469.00 & $\begin{array}{l}16383.0 \\
0\end{array}$ & 5584.00 & 5750.00 & 6622.00 & 425704.00 & 10732.00 \\
\hline Egbelu & 0.045 & 3067.00 & $\begin{array}{l}19644.0 \\
0\end{array}$ & 6516.00 & 7011.00 & 8208.00 & 402484.00 & 15095.00 \\
\hline Adageorge & 0.01 & 642.00 & 1851.00 & 1293.00 & 745.00 & 1589.00 & 410730.00 & 2959.00 \\
\hline
\end{tabular}

\begin{tabular}{|c|c|c|c|c|c|c|c|c|c|c|}
\hline & $\begin{array}{l}\text { Weight } \\
\text { of } \\
\text { particul } \\
\text { ate } \\
\text { matter }\end{array}$ & $\begin{array}{l}(25) \\
\mathrm{Sb} \\
121\end{array}$ & $\begin{array}{l}(26) \\
\text { Ba } \\
137\end{array}$ & $\begin{array}{l}(27) \\
\text { Tb } \\
159\end{array}$ & $\begin{array}{l}(\mathbf{2 8}) \\
\mathbf{L u} \\
\mathbf{1 7 5}\end{array}$ & $\begin{array}{l}(29) \\
\text { Tl / } \\
205\end{array}$ & $\begin{array}{l}(30) \\
\text { Pb } \\
208\end{array}$ & $\begin{array}{l}\text { (31) } \\
\text { Bi / } 209\end{array}$ & $\begin{array}{l}(32) \\
\text { Th / } \\
232\end{array}$ & $\begin{array}{l}(33) \\
\mathbf{U} \\
238\end{array}$ \\
\hline $\begin{array}{l}\text { Sampling } \\
\text { points }\end{array}$ & {$[\mathrm{g}]$} & $\begin{array}{l}\text { Conc. } \\
{\left[\begin{array}{ll}{[\quad \mu \mathrm{g} /} \\
\left.\mathrm{m}^{3}\right]\end{array}\right.}\end{array}$ & $\begin{array}{l}\text { Conc. } \\
{\left[\begin{array}{ll}{[\quad \mu g /} \\
\left.\mathrm{m}^{3}\right]\end{array}\right.}\end{array}$ & $\begin{array}{l}\text { Conc. } \\
{\left[\begin{array}{ll}{[\quad \mu g /} \\
\left.\mathrm{m}^{3}\right]\end{array}\right.}\end{array}$ & 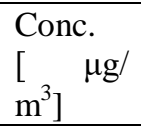 & $\begin{array}{l}\text { Conc. } \\
{[\mu \mathrm{g} /} \\
\left.\mathrm{m}^{3}\right] \\
\end{array}$ & $\begin{array}{l}\text { Conc. } \\
{\left[\begin{array}{ll}{[\quad \mu g /} \\
\left.\mathrm{m}^{3}\right]\end{array}\right.}\end{array}$ & $\begin{array}{l}\text { Conc. } \\
{\left[\begin{array}{ll}{[} & \mu \mathrm{g} / \\
\left.\mathrm{m}^{3}\right]\end{array}\right.}\end{array}$ & $\begin{array}{l}\text { Conc. } \\
{\left[\begin{array}{l}\left.\mathrm{m}^{3}\right] \\
\mathrm{g} / \mathrm{g} /\end{array}\right.}\end{array}$ & $\begin{array}{l}\text { Conc. } \\
{\left[\begin{array}{l}{ }^{3}{ }^{3} \\
\mathrm{~m}^{2}\end{array}\right.}\end{array}$ \\
\hline $\begin{array}{l}\text { Rumuolu } \\
\text { meni }\end{array}$ & 0.037 & $\begin{array}{l}254906 . \\
00\end{array}$ & $\begin{array}{l}169330 . \\
00\end{array}$ & $\begin{array}{l}472138 . \\
00\end{array}$ & $\begin{array}{l}427900 . \\
00\end{array}$ & $\begin{array}{l}718.9 \\
1\end{array}$ & $\begin{array}{l}440700 . \\
00\end{array}$ & $\begin{array}{l}209018 . \\
00\end{array}$ & $\begin{array}{l}28792 . \\
92\end{array}$ & $\begin{array}{l}17265 . \\
42\end{array}$ \\
\hline Egbelu & 0.045 & $\begin{array}{l}241563 . \\
00\end{array}$ & $\begin{array}{l}141382 . \\
00\end{array}$ & $\begin{array}{l}442908 . \\
00\end{array}$ & $\begin{array}{l}403222 . \\
00\end{array}$ & $\begin{array}{l}525.5 \\
7\end{array}$ & $\begin{array}{l}596995 . \\
00\end{array}$ & $\begin{array}{l}212726 . \\
00\end{array}$ & $\begin{array}{l}32393 . \\
68\end{array}$ & $\begin{array}{l}10067 . \\
36\end{array}$ \\
\hline Adageorge & 0.01 & 1589.00 & $\begin{array}{l}125817 . \\
00\end{array}$ & $\begin{array}{l}425468 . \\
00\end{array}$ & $\begin{array}{l}395629 . \\
00\end{array}$ & 98.52 & $\begin{array}{l}76811.0 \\
0\end{array}$ & $\begin{array}{l}225859 . \\
60\end{array}$ & $\begin{array}{l}2844.7 \\
6\end{array}$ & $\begin{array}{l}1010.7 \\
9\end{array}$ \\
\hline
\end{tabular}

\subsection{Health Implication of Result}

Some metals have been identified as very essential to plants and animals. According to Tchounwou et aal, (2014) metals such as cobalt $(\mathrm{Co})$, copper $(\mathrm{Cu})$, chromium $(\mathrm{Cr})$, iron $(\mathrm{Fe})$, magnesium (Mg), manganese (Mn), molybdenum (Mo), nickel (Ni), selenium (Se) and zinc ( $\mathrm{Zn}$ ) are essential nutrients that are required for various biochemical and physiological functions, but when their supply are not sufficient, it results in a variety of medical syndrome. However, some 
heavy metals such as copper $(\mathrm{Cu})$, selenium, and zinc at higher concentrations can lead to poisoning, and for short periods of exposure to high levels of antimony( $\mathrm{Sb})$ induces nausea, vomiting, and diarrhoea (Lenntech, 2017). Research studies have shown a link between aluminium(Al) accumulation in the and Alzheimer's disease (a degenerative disorder of the brain that leads to dementia), and Parkinson disease (a gradual nervous disorder characterized by symptoms of trembling hands, lifeless face, monotone voice, and a slow shuffling walk)( Global Healing Center, 2015). Lead $(\mathrm{Pb})$ causes disorder of the nervous system and anaemia; nickel (Ni) causes cancer of the lungs and skin; tin ( $\mathrm{Sn})$ causes central nervous system disorder, visual impairment and pneumoconiosis; manganese (Mn) affects the nervous system; chromium $(\mathrm{Cr})$ induces respiratory cancers; arsenic (As) causes respiratory cancer and perforation of nasal septum (Mahurpawar, 2015). Thorium (Th) and Uranium (U) are examples of actinides. According to Helmenstine (2017), all actinides are radioactive. These actinides have less effect when they are ingested, but more damaging when they are inhaled as they remain in the lungs and irradiate the lung tissue (wikipedia). Potassium (K) inhalation causes irritation of the nose, eyes, throat, lungs with coughing and at a higher concentration may result to build of fluid in the lungs which could lead to death; Silver ( $\mathrm{Ag}$ ) inhalation causes dizziness, breathing difficulty and state of coma or death when its concentration is high; Bismuth (Bi) causes irritation of the respiratory tracts (Lenntech, 2017). World Health organisation (WHO, 2000) report says vanadium (V) has increased in recent years due to crude oil residue in power plant and describes its inhalation as a potent respiratory irritant. Beryllium is harmful when it is breathe into the nostril. According to Agency for Toxic Substances and Disease Registry (ATSDR, 2002), inhaling large amounts of soluble beryllium compounds (greater than $1 \mathrm{mg}$ beryllium per cubic meter of air, $1 \mathrm{mg} / \mathrm{m}^{3}$ ), causes pneumonia with reddening and swelling of the lungs. Inhalation of cadmium $(\mathrm{Cd})$ is also suspected to be responsible for lung cancer, chronic rhinitis, destruction of the olfactory epithelium with subsequent anosmia and development of bronchitis (ATSDR, 2013). For Beryllium (Be), Chronic Beryllium Disease (CBD) is a chronic granulomatous lung disease caused by inhaling airborne beryllium and is characterised by shortness of breath, unexplained coughing, fatigue, weight loss, fever, and night sweats(United States Department for Labour, n.d). The breathing organs, skin and the digestive tract absorb thallium (Tl) very effectively, but it poisoning is mainly caused by accidental uptake of rat poison that causes stomach aches and nervous system damage (Geiger \& Cooper, 2010). Sodium (Na) forms sodium hydroxide $(\mathrm{NaOH})$ fumes in the presence of water, which are irritating to the nose and in severe case, is capable of causing breathing difficulty, coughing and chemical bronchitis (Lenntech 2017).

\section{Conclusion}

The masses of particulate matter collected from two construction sites (sampling sites 1 and 2) and a residential building (sampling site 3 ) for a period of three weeks and two days (April $19^{\text {th }}-$ May $10^{\text {th }}, 2017$ ) were $0.06 \mathrm{~g}$ and $0.04 \mathrm{~g}$ for sampling sites 1 and 2 , while sampling site 3 being the control was $0.02 \mathrm{~g}$. The Atomic Absorption Spectroscopic analyses of the particulate matter samples revealed the presence of thirty three (33) elemental components which include; beryllium $(\mathrm{Be})$, sodium $(\mathrm{Na})$, magnesium $(\mathrm{Mg})$, aluminium $(\mathrm{Al})$, potassium $(\mathrm{K}), \operatorname{calcium}(\mathrm{Ca})$, vanadium(V), chromium (Cr 52 \& Cr 53), manganese(Mn), iron (Fe), Cobalt(Co), nickel(Ni), copper( $\mathrm{Cu})$, zinc(Zn), arsenic(As), molybdenum(Mo 95,\& Mo 98), cadmium(Cd106,Cd108 \& 
Cd 111), Silver(Ag), indium(In), tin(Sn), antimony (Sb), barium(Ba), terbium(Tb), thallium(Tl), lead $(\mathrm{Pb})$, bismuth(Bi), thorium(Th), and uranium(U).

A good number of research work have shown that some of these elements constitute serious health problems to man when they are inhaled or ingested, hence the need for proper safety mechanisms to be put in place in order to protect construction workers and individuals living within the environment from their harmful effects.

\section{References}

[1] Agency for Toxic Substances and Disease Registry. (ATSDR, 2002). Public Health Statement for Beryllium. Retrieved December 20th, 2017 from https://www.atsdr.cdc.gov/csem/csem.asp?csem $=6 \& p o=12$

[2] Agency for Toxic Substances and Disease Registry (ATSDR 2013). Case Studies in Environmental Medicine (CSEM) Cadmium Toxicity. Retrieved December 20th, 2017 from https://www.atsdr.cdc.gov/csem/csem.asp?csem $=6 \& p o=12$

[3] Europeans Environment Agency. (2012). Particulate from natural sources and related reporting under the EU Air Quality Directive in 2008 and 2009. Retrieved December 11th, 2017 from https://www.eea.euroa.eu

[4] Geiger, A \& Cooper, J. (2010). Overview of Airborne Metals Regulations, Exposure Limits, Health Effects, and Contemporary Research, Cooper Environmental Services, LCC, Portland.

[5] Gray, J. (2017). Pollution from construction. Retrieved April1st, 2017 from www.sustainablebuiltco.uk/pollutionfromconstruction.html.

[6] Green Facts. (2017). Air Pollution Particulate Matter. Retrieved from https://www.greenfacts.org/en/particulate-matter-pm/level-2/01-presentation.htm

[7] Global Healing Center. (2015), the Effects of Toxic Metals. Retrieved December 6th, 2017 from https://www.globalhealingcenter.com/natural-health/effects-of-toxic-metals/

[8] Harvey, C. (2015). Energy and Environment. The Washington post. Retrieved from htts:www.washingpool.community.com/new/energynvironment.

[9] Helmenstine, M.A. (2017). Actinides (Actinide Series), Properties and Reactions of the Actinide Series of Elements. Retrieved December 8th, 2017 fromhttps://www.thoughtco.com/actinides606643.

[10] Lenntech, 2017. Heavy Metals. Retrieved November 28th, 2017 from https://www.lenntech.com/processes/heavy/heavy-metals/heavy-metals.htm

[11] Lenntech (2017) Chemical properties of potassium - Health effects of potassium - Environmental effects of potassium Retrieved December13th, 2017 from https://www.lenntech.com/periodic/elements/k.htm.

[12] Lenntech (2017). Chemical properties of sodium - Health effects of sodium - Environmental effects of sodium. Retrieved December 22nd, 2017 from https://www.lenntech.com/periodic/elements/k.htm.

[13] Tchounwou, P.P., Yedjou, C. G, Patlolla, A. k., Sutton, D. J (2012). Heavy Metals Toxicity and the Environment. NIH-RCMI Center for Environmental Health, College of Science, Engineering and Technology, Jackson State University, USA.

[14] TaekRim, K., HoKoo, K., SunPark J. (2013). Toxicological Evaluations of Rare Earths and Their Health Impacts to Workers: A Literature Review. Safety and Health at Work, Elsevier Korea LLC.

[15] TutorVista.com. (2017). Atomic Absorption Spectroscopy. Retrieved November 22end, 2017 from chemistry.tutorvista.com

[16] The National Institute for Occupational Safety and Health (NIOSH, 2015). Indium Retrieved December 21st, 2017 from https://www.cdc.gov/niosh/ipcsneng/neng1293.html 
[17] WHO. (2000). Air Quality Guidelines. Regional Office for Europe, Copenhagen, Denmark, 2000

[18] Loomis, D., Huang, W., and Chen, G.(2014). The International Agency for Research on Cancer (IARC) evaluation of the Carcinogenicity of Outdoor air Pollution: Focus on China. Chinese Journal of Cancer. 33(4), pp89-196.

[19] LTS Research Laboratories. (2015). Safety Data Sheet Terbium Oxide Retrieved December 12th, 2017 from https://www.ltschem.com/msds/Tb2O3.pdf.

[20] Mahurpawar, M. (2015). Effects of Heavy Metals on Human Health. International Journal of Research- Granthaalayah. Social Issues and Environmental Problems: September, 2015]; PP 1 -7

[21] McCain, M. (1997). Chemtrails and Barium Toxicity. Rense.com. Retrieved December 21st, 2017 from http://www.rense.com/general21/tox.htm

[22] Morawska, L. (1999). Environmental Aerosol Physics. Queensland University of Technology Bribane, Australia.

[23] PMCID: PMC4144270

[24] United States Department for Labour. (n.d). Occupational Safety and Health Administration. Retrieved December 12th, 2017 from https://www.osha.gov/SLTC/beryllium/healtheffects.html

[25] USEPA, s(2017). Particulate Matter (PM) Pollution. Retrieved July 11th, 2017 from www.epa.gov/pm-pollution.

\footnotetext{
*Corresponding author.

E-mail address: drobijames@yahoo.com
} 